\title{
Correction to: The U\&I study: study protocol for a feasibility randomised controlled trial of a pre-cognitive behavioural therapy digital 'informed choice' intervention to improve attitudes towards uptake and implementation of CBT for psychosis
}

Kathryn Greenwood ${ }^{1,2^{*}}$, Katie Alford ${ }^{3}$, lain O'Leary ${ }^{1}$, Emmanuelle Peters ${ }^{4,5}$, Amy Hardy ${ }^{4,6}$, Kate Cavanagh ${ }^{2}$, Andy P. Field ${ }^{2}$, Richard de Visser ${ }^{2}$, David Fowler ${ }^{1,2}$, Matthew Davies ${ }^{1}$, Alexandra Papamichail ${ }^{1}$ and Philippa Garety ${ }^{4,6}$

\section{Correction to: Trials}

https://doi.org/10.1186/s13063-018-3023-7

Following publication of the original article [1], the authors reported a typing mistake in the spelling of author Iain O'Leary. The original article has been corrected.

In this Correction the incorrect and correct spellings are shown.

Incorrect spelling:

- Ian O’Leary

The correct spelling is:

- Iain O'Leary

\section{Author details}

${ }^{1}$ R\&D Department, Sussex Partnership NHS Foundation Trust, Sussex Education Centre, Millview Hospital Site, Nevill Avenue, Hove BN3 7HZ, UK. ${ }^{2}$ School of Psychology, University of Sussex, Pevensey Building, Falmer, Brighton, East Sussex BN1 9RP, UK. ${ }^{3}$ Brighton and Sussex Medical School, University of Sussex, Falmer, Brighton BN1 9RP, UK. ${ }^{4}$ Department of Psychology, Institute of Psychiatry, Psychology and Neuroscience, Kings College London, De Crespigny Park, Denmark Hill, London SE5 8AF, UK. ${ }^{5}$ PICuP Clinic, South London and Maudsley NHS Foundation Trust, Maudsley Hospital, Denmark Hill, London SE5 8NZ, UK. ' ${ }^{6}$ South London and Maudsley NHS Foundation Trust, Bethlem Royal Hospital, Monks Orchard Road, Beckenham, Kent BR3 3BX, UK.
Received: 27 November 2018 Accepted: 27 November 2018 Published online: 12 December 2018

\section{Reference}

1. Greenwood K, et al. The U\&l study: study protocol for a feasibility randomised controlled trial of a pre-cognitive behavioural therapy digital 'informed choice' intervention to improve attitudes towards uptake and implementation of CBT for psychosis. Trials. 2018;19:644. https://doi.org/10. 1186/s13063-018-3023-7.

* Correspondence: k.e.greenwood@sussex.ac.uk

${ }^{1}$ R\&D Department, Sussex Partnership NHS Foundation Trust, Sussex Education Centre, Millview Hospital Site, Nevill Avenue, Hove BN3 7HZ, UK

${ }^{2}$ School of Psychology, University of Sussex, Pevensey Building, Falmer, Brighton, East Sussex BN1 9RP, UK

(c) The Author(s). 2018 Open Access This article is distributed under the terms of the Creative Commons Attribution 4.0 International License (http://creativecommons.org/licenses/by/4.0/), which permits unrestricted use, distribution, and reproduction in any medium, provided you give appropriate credit to the original author(s) and the source, provide a link to the Creative Commons license, and indicate if changes were made. The Creative Commons Public Domain Dedication waiver (http://creativecommons.org/publicdomain/zero/1.0/) applies to the data made available in this article, unless otherwise stated. 